\title{
Dehydroepiandrosterone Supplementation and the Impact of Follicular Fluid Metabolome and Cytokinome Profiles in Poor Ovarian Responders
}

\author{
Veronique Viardot-Foucault \\ KK Women's and Children's Hospital \\ Jieliang Zhou \\ KK Women's and Children's Hospital \\ Dexi Bi
}

Tongji University

Yoshihiko Takinami

Bruker, Japan

Jerry.K.Y. Chan

KK Women's and Children's Hospital

Yie Hou Lee ( $\square$ yiehou.lee@smart.mit.edu )

SingHealth Duke-NUS Academic Medical Centre

\section{Research Article}

Keywords: metabolomics, follicular fluid, DHEA, poor ovarian responder, cytokines

Posted Date: February 7th, 2022

DOI: https://doi.org/10.21203/rs.3.rs-1314035/v1

License: (1) This work is licensed under a Creative Commons Attribution 4.0 International License.

Read Full License 


\section{Abstract}

Background: Poor ovarian responders (POR) are women undergoing in-vitro fertilization who respond poorly to ovarian stimulation, resulting in the retrieval of lower number of oocytes, and subsequently lower pregnancy rates. The follicular fluid (FF) provides a crucial microenvironment for the proper development of follicles and oocytes through tightly controlled metabolism and cell signaling. Conversely, dysregulated FF metabolism and cytokine production in POR could impart or reflect detrimental effects on oocytes. Androgens such as dehydroepiandrosterone (DHEA) have been proposed to alter the POR follicular microenvironment and induce metabolic changes but its utility in improving pregnancy rates remain uncertain, partially due to the unknown impact DHEA imposes on the FF metabolome and cytokine profiles.

Methods: FF samples were collected from 52 POR patients who underwent IVF with DHEA supplementation (DHEA+) and without (DHEA-). The FF samples were split for profiling analyses using untargeted liquid chromatography-tandem mass spectrometry (LC-MS/MS) metabolomics and a largescale multiplex suspension immunoassay covering 65 cytokines, chemokines and growth factors. Multivariate statistical modelling by partial least squares-discriminant regression (PLSR) analysis was performed for revealing metabolome-scale differences. Further, differential metabolite analysis between the two groups was performed by PLSR $\beta$-coefficient regression analysis and Student's t-test.

Results: Untargeted metabolomics identified $118 \mathrm{FF}$ metabolites of diverse chemistries and concentrations which spanned three orders of magnitude. They include metabolic products highly associated with ovarian function - amino acids for regulating $\mathrm{pH}$ and osmolarity, lipids such fatty acids and cholesterols for oocyte maturation, and glucocorticoids for ovarian steroidogenesis. The FF metabolomic profiles of DHEA+ and DHEA- groups were notably distinct as classified by multivariate partial least squares regression analysis. Specifically, glycerophosphocholine, linoleic acid, progesterone, and valine were significantly lower in DHEA+ relative to DHEA-. Using the large-scale cytokine profiling assay, we observed significantly lower MCP1, IFNy, LIF and VEGF-D levels in DHEA+ relative to DHEA.

Conclusions: Collectively, our large-scale study characterized a diversity of metabolites and cytokines in the FF of POR individuals and identified several of these compounds associated DHEA supplementation.

\section{Background}

Poor ovarian responders (POR) are a sub-group of infertile women that account for $9-26 \%$ of in vitro fertilization (IVF)indications. (1,2)In patients designatedas "poor responders," so-called due to poor response to ovarian stimulation given during IVF workup, the limited number of obtained oocytes remains the major problem in optimizing the live birth rates.(3)As a result of a lower number of oocytes retrieved, there are fewer embryos to select and transfer, and subsequently these patients have lower pregnancy rates per transfer and lower cumulative pregnancy rates per started cycle compared with normal responders. In PORs the mechanism of ovarian insufficiency can be multifactorial with causes such as 
ovarian surgery especially in case of endometrioma, $(4,5)$ uterine artery embolization for the treatment of uterine leiomyoma, $(6,7)$ genetic defects, chemotherapy, radiotherapy, autoimmune disorders, single ovary, chronic smoking, $(8,9)$ or linked to diseases such as diabetes mellitus Type I.(10)However, in most cases, follicular depletion plausibly reflecting premature ovarian aging,(11) clinically translates into a reduction of implantation rates, an increase of early pregnancy loss, and disappointingly low IVF success.(12,13)

In each menstrual cycle, human ovaries produce a single dominant follicle. Growth of the dominant follicle encompasses enlargement of the oocyte, replication of follicular cells, and formation and expansion of a fluid-filled follicular antrum or cavity, providing a specialized microenvironment for the development of oocytes. Follicular fluid (FF) that fills the antrum cavity is derived from the surrounding theca capillaries, abundant and easily accessible during IVF procedures due to ample volume being produced during follicle maturation.(14) FF are rich in metabolites, notably hormones, amino acids and lipids that are critical for oocyte growth and development, which determines subsequent potential to achieve fertilization and embryo development.(15) As such, constituents of the FF surrounding the oocyte provides a unique biochemical windowto the growth and differentiation of the oocyte.(16)To unravel the biochemical composition of human FF and its impact on oocyte development, metabolomic analyses using gas chromatography-mass spectrometric and proton nuclear magnetic resonance metabolomic analyses have been conducted,(17-21) as were proteomic analyses.(22-27)These studies mainly report the FF profiles of IVF patients, whereas the FF metabolome of POR remains poorly characterized. Furthermore, the effect of dehydroepiandrosterone (DHEA) on the FF metabolome has not been previously studied.

DHEA is a steroid produced in the adrenal cortex and the ovarian theca cells in women that is converted into more active forms of androgens such as testosterone.(5) It has been suggested that DHEA supplementation may increase the number of available follicles in PORsthrough an increased serum level of insulin-like growth factor, increased follicular response to follicle stimulating hormone (FSH), shifts to aerobic metabolism (28)and improved quality of oocytes.(29) However, the efficacy of DHEA pretreatment has been controversial, with partial to reasonable clinical evidence being observed(3034). Based on these findings, we conducted a study to evaluate whether the FF metabolome differed in POR patientstreated with DHEA or not, and whether the FF metabolome may be predictive of IVF outcome. Furthermore, DHEA has immunoregulatory functions,(35) and a large-scale study of FF cytokines was conducted in parallel to reveal DHEA immunomodulatory targets.

\section{Material And Methods}

\section{Ethical approval and study population}

The local Institutional Review Board approved the study (CIRB/2011/404/D) and written informed consent was obtained from each participant. A case-control study was conducted to evaluate the metabolic and cytokine effect of DHEA administration in women, below the age of 42 starting their IVF treatment who met one of the two following features of POR (an abnormal ovarian reserve test and/or a 
previous poor response to ovarian stimulation in an IVF cycle) were assessed for eligibility.(3)The ESHRE working group on Poor Ovarian Response Definition of diminished ovarian reserves (AMH $<1.0 \mathrm{ng} / \mathrm{mL}$ or Day 2 or $3 \mathrm{FSH}>10 \mathrm{IU} / \mathrm{L}$ ), or women with fewer than four oocytes retrieved with either standard long or antagonist protocols was used to defined POR in this study(3).Inclusion criteria included women with diminished ovarian reserves (anti-müllerian hormone $<1.0 \mathrm{ng} / \mathrm{mL}$ or D2/3 follicle stimulating hormone $>10$ IU/L), or women with fewer than four oocytes retrieved with either standard long or antagonist protocols. The study excluded women with previous or current DHEA supplementation, use of corticosteroids within the past three months, major systemic illnesses, and allergy to DHEA. 28 eligible patients received DHEA (Pharma Natural, USA) at the dose of $75 \mathrm{mg} /$ day for three to eight months prior starting their controlled ovarian stimulation (COS), herein known as DHEA+, and 24 patients received no treatment (DHEA-). Table 1summarizes the baseline characteristics of the patients in this study.Average age (DHEA-, 36 years; DHEA,+ 37 years) and body mass index (DHEA-, $24 \mathrm{~kg} / \mathrm{m}^{2}$; DHEA,$+ 23 \mathrm{~kg} / \mathrm{m}^{2}$ ) were similar in both groups $(p>0.05)$. Baseline hormones between the two groups were comparable.

\section{IVF/ICSIprotocol}

All individuals received the same stimulation protocol, same starting dose of gonadotropin, and fertilization technique. Briefly, the IVF/ICSI treatment cycle involved an antagonist-based COS protocol consisting of daily sub-cutaneous injections of recombinant-FSH (Puregon, Follitropin beta, 300iu; MSD, USA) and highly-purified human menopausal gonadotropin (Menopur; Menotropin, 150 IU; Ferring Pharmaceuticals, Germany) with initiation of gonadotropin releasing hormone antagonist (Ganirelix, Orgalutan, $0.25 \mathrm{mg} \mathrm{s} / \mathrm{c}$; MSD, USA) on day 5 of COS. The dose of Menopur and Puregon could be further increased depending on individual ovarian response. All patients had thisstandardized antagonist (short) protocol: no agonist (long) protocol was used. Human chorionic gonadotropin (i.m 10,000IU hCG; Pregnyl; MSD, USA) was administered when at least one follicle measured $\geq 17 \mathrm{~mm}$ in diameter (averaged orthogonal measurements). The endometrial thickness, peak estradiol and progesterone levels were assessed on the day of human chorionic gonadotropin (hCG) trigger. Ultrasound-guided trans-vaginal oocyte retrieval was performed 36 hours after $\mathrm{hCG}$ administration. The effect of DHEA supplementation on the markers of ovarian reserve (anti-müllerian hormone; AMH), and follicular function (IGF-1)(36), as well as ovarian follicular levels of estradiol, testosterone, and DHEA collected from the lead follicle at the time of OPU were assessed through ELISAas previously described(37).

Embryo transfer was performed on day 2 or day 3 of embryo-culture, and luteal phase support was achieved with vaginal progesterone (micronized progesterone, Utrogestan, $200 \mathrm{mg}$ three times a day, Besins-International, France). Pregnancy was established by serum beta-hCG seventeen days post embryo transfer. Clinical pregnancy will be established by a transvaginal ultrasound four weeks after embryo transfer.

\section{Sample preparation}


FF (DHEA+, $N=18$ and DHEA-, $N=16)$ were divided for metabolomics and cytokine analyses. For untargeted metabolomics analysis, sample preparation followed previously published reports with some modifications.(38,39) A volume of $50 \mu \mathrm{L}$ from each FF sample was thawed at $4^{\circ} \mathrm{C}$, and FF proteins were precipitated with $200 \mu \mathrm{L}$ ice-cold methanol. After vortexing, the mixture was centrifuged at 16,000 rpm for $10 \mathrm{~min}$ at $4^{\circ} \mathrm{C}$ and the supernatant was collected and evaporated to dryness in a speedvacuum evaporator. The dry extracts were then re-dissolved in $200 \mu \mathrm{L}$ of water/methanol (98:2; v/v) for liquid chromatography-tandem mass spectrometry (LC-MS/MS) analysis.

A pooled quality control (QC) sample was generated to allow comparison of analytic behavior over long periods of time. The pooled reference samples were for the purposes of quality control (i.e., to ensure relative consistency among identical samples within days) and for quality assurance (i.e., to ensure consistent results between days). They did not contribute data to downstream statistical analysis.

\section{Liquid Chromatography-Tandem Mass Spectrometry-based Metabolomics}

The supernatant fraction from sample preparation step was analyzed using Agilent 1290 ultra-high pressure (performance) liquid chromatography system (Waldbronn, Germany) equipped with a Bruker impact II Q-TOF mass spectrometer with its normal electrospray ionization (ESI) ion source (Bruker Daltonics). $2.5 \mu \mathrm{L}$ of samples was injected and were separated using Waters Acquity HSS T3 (2.1 mm i.d. $x 100 \mathrm{~mm}, 1.8 \mu \mathrm{m}$ ) at a flow rate of $0.2 \mathrm{~mL} / \mathrm{min}$. The oven temperature was set at $50^{\circ} \mathrm{C}$. The gradient elution involved a mobile phase consisting of (A) $0.1 \%$ formic acid in water and (B) $0.1 \%$ formic acid in methanol. The initial condition was set at 5\% B. A 5.5 min linear gradient to $60 \%$ B was applied, followed by a 13 min gradient to $98 \%$ B (total 24 min including wash and re-equilibration) at a flow rate of 0.4 $\mathrm{ml} / \mathrm{min}$. The ion spray voltage was set at 4,500V, and the DryTemperature was maintained at ${ }^{\circ} \mathrm{C}$. The drying nitrogen gas flowrate and the nebulizer gas pressure were set at $8.0 \mathrm{~L} / \mathrm{min}$ and 26 psi, respectively. Calibration of the system was performed using sodium formate clusters before data acquisition. The stability of the LC-MS method was examined and evaluated by sodium formate clusters $(1 \mathrm{mM} \mathrm{NaOH}$, $0.1 \%$ formic acid, 50\% 2-propanol) infused into the system.

The ESI mass spectra were acquired in positive ion mode. Mass data were collected between $\mathrm{m} / \mathrm{z} 100$ and 1000 at a rate of three scans per second. Auto MS/MS was triggered at $8 \mathrm{~Hz}$ with duty cycle of $1.5 \mathrm{~s}$. Threshold was set at 1500 counts, with active exclusion activated after 3 spectra, released after 0.3 min and overwritten if the current or previous intensity changes. MS/MS spectra were acquired at collision energyof 20-50 eV automatically varied by the charge states and the intensities of the selected precursors. Fragment spectra acquisition was carried out at a scan rate dependent on the MS precursor intensities - MS/MS spectra for high-intensity precursors were acquired for a shorter time (90000 counts, $12 \mathrm{~Hz}$ ) than low-intensity precursor ions $(10000$ counts, $6 \mathrm{~Hz}$ ) thus allowing for a balancing of maximal scan time and MS/MS spectral quality. As shown in Figure S1, the eight pooled quality control samples clustered in Principal Component Analysis (PCA) scores plots, and together with retention time CV\% $<0.1$ min, peak $m / z$ values $3 \mathrm{mDa}$, and relative standard deviations of peak areas $<20 \%$, there was good system stability, mass accuracy and reproducibility of the chromatographic separation during the whole 
LC-MS/MS sequence. In addition, intensity $\mathrm{CV} \%$ of the identified compounds in pooled quality control samples are low (average 6\%). PCA hotelling $\left(\mathrm{T}^{2}\right)$ revealed one DHEA+ subject as an outlier (D4) and was removed from further analysis (Figure S1).

\section{Compound identification}

Structure identification was achieved via the following in MetaboScape (version 2.0): elemental composition was predicted via isotopic pattern following the rules (i) mSigma of MS1: 20 with tolerance of $5 \mathrm{ppm}$ and (ii) MS2: 50 with tolerance of $2 \mathrm{mDaof}$ the differential metabolites was searched against Bruker HMDB (Human Metabolome Database) using a precursor match of $\pm 10 \mathrm{mDa}$, minimum score of 400 and minimum match score of 250 . Progesterone, glycerophosphocholine, linoleic acid and valine were structurally confirmed using chemical standards.

\section{Multiplex immunoassay analysis}

45 cytokines were detected and measured using ProCartaplex (EBioscience, CA, USA) as previously reported [BDNF, EGF, Eotaxin (CCL11), FGF-2 (FGF basic), GM-CSF, CXCL1 (GROa), HGF, IFNY, IFNa, IL-1RA, IL-1 , IL-1a, IL-2, IL-4, IL-5, IL-6, IL-7, CXCL8 (IL-8), IL-9, IL-10, IL-12 p70, IL-13, IL-15, IL-17A, IL-18, IL-21, IL22, IL-23, IL-27, IL-31, CXCL10 (IP-10), LIF, CCL2 (MCP-1), CCL3 (MIP-1a), CCL4 (MIP-1ß), BNGF, PDGF-BB, PLGF, CCL5 (RANTES), SCF, CXCL12 (SDF1a), TNFa, LTA (TNFß), VEGF-A, VEGF-D].(40)Briefly, $5 \mu$ L of FFs were diluted with $5 \mu \mathrm{L}$ Universal Dilution Buffer, and mixed with $50 \mu \mathrm{L}$ of antibody-conjugated, magnetic beads in a 96 well DropArray plate (Curiox Biosystems, Singapore) and rotated at $450 \mathrm{rpm}$ for $120 \mathrm{~min}$ at $25^{\circ} \mathrm{C}$ while protected from light. Beads were internally dyed with different concentrations of two spectrally distinct fluorophores and covalently conjugated to antibodies against the 45 cytokines, chemokines and growth factors. The plate was washed three times with wash buffer (PBS, 0.05\% Tween-20) on the LT210 Washing Station (Curiox) before adding $10 \mu \mathrm{L}$ of secondary antibody and rotating at $450 \mathrm{rpm}$ for $30 \mathrm{~min}$ at $25^{\circ} \mathrm{C}$ protected from light. Subsequently, the plate was washed three times with wash buffer, and $10 \mu \mathrm{L}$ of streptavidin-phycoerythrin added and rotated at $450 \mathrm{rpm}$ for $30 \mathrm{~min}$ at $25^{\circ} \mathrm{C}$ protected from light. The plate was again washed thrice with wash buffer; $60 \mu \mathrm{L}$ of reading buffer was then added and the samples read using the Bio-Plex Luminex 200 (BioRad). The beads were classified by the red classification laser $(635 \mathrm{~nm})$ into its distinct sets, while a green reporter laser ( $532 \mathrm{~nm})$ excites the phycoerythrin, a fluorescent reporter tag bound to the detection antibody. Quantitation of the 45 cytokines was then determined by extrapolation to a six or seven-point standard curve using five-parameter logistic regression modelling. Calibrations and validations were performed prior to runs and on a monthly basis respectively.

\section{Statistical analysis}

GraphPad Prism 6 (GraphPad Software Inc.) was used for performing all statistical analyses. Data were checked for normal distribution using Kolmogorov-Smirnov test. Unpaired or paired t-test was performed, as appropriate, to determine statistical significance between groups form normally distributed data. Mann-Whitney U test was used for non-normally distributed data. For comparing more than three groups, 
the data were analyzed using ANOVA test, followed by the $t$-test with Bonferroni adjustment. $P<0.05$ was considered significant.Metabolomic data was further analyzed by Principal Component Analysis (PCA) and Partial Least Squares Regression (PLSR) modelling (Unscrambler X version 10.1) after the normalization of data by first centering the data to the median and scaling it by division with the standard deviation. Full cross-validation was applied in PLSR to increase model performance and for the calculation of $\beta$-coefficient regression values. Metabolites with $\beta$-coefficient regression values $\geq 1$ are considered to have contribute significantly to the PLSR model. In this study, metabolites fulfilling both PLSR $\beta$-coefficient regression values $>1.2$ and Student t-test $p<0.05$ was considered as differential.

\section{Results}

\section{Clinical observations}

IVF/ICSI clinical and hormonal outcomes are shown in Table 2. There was no significant difference in clinical pregnancy, number of oocytes retrieved, metaphase II oocytes or number of embryos transferred. Significant increases were observed for clinical hormonal markers DHEA-S and free testosterone in the DHEA+ patients compared to the DHEA- patientswith treatment ( $p<0.001$; Figure S4). Additionally, estradiol,freetestosterone and DHEA-S were significantly higher 4 months after treatment in the DHEA+ patients $(p<0.001$; Table 3 )

\section{Follicular fluid metabolomic analysis of POR patients}

From a total 2717 time-aligned features, an average of 903 features was chosen for auto MS/MS mode. From these, a total of 100 metabolites were identified via chemical standard confirmed HMDB(41).An average of $65 \mathrm{MS} / \mathrm{MS}$ confirmed metabolites was identifiedper patient, which was similar in terms of metabolite identified in either DHEA- or DHEA+ subjects ( $p=0.8$; range:59-76 metabolites), providing a global metabolome view of the FF metabolome in POR patients (Table S1). The FF metabolomespanned three orders of magnitude, and was composed of a range of chemically diverse metabolites including, glycerophospholipids and derivatives (glycerophosphocholine, phosphatidylcholines), fatty acids (heptadecanoic acid, linoleic acid, vaccenic acid, myristic acid), cholesterols (isocaproic acid, 7ketocholesterol), glucocorticoids (11-deoxycortisol or cortexolone, cortisol, corticosterone), hormones (17hydroxyprogesterone, deoxycorticosterone, 11a-hydroxyprogesterone, 16-dehydroprogesterone, androstenedione, epitestosterone, progesterone, pregnenolone). Other metabolites includedbile acids (3bhydroxy-5-cholenoic acid, 3-oxocholic acid, glycocholic acid), peptides and derivatives (3-indolepropionic acid), lactones (delta-hexanolactone/caprolactone), lactic acid, vitamin D3 and sphingosine (Table 4).

\section{Altered POR follicular fluid metabolome in response to DHEA}

Next, partial least squares-discriminant regression analysis (PLSR) was performed based on the overall features to compare the FF metabolomic profiles between the DHEA+ and DHEA- groups. At metabolomescale, the PLSR score plot showed that the FF metabolome clearly distinguished DHEA+ patients from DHEA- patients (Figure 1). Further analysis revealed that in DHEA- subjects, progesterone was the most 
abundant FF metabolite, followed by L-alanine, L-phenylalanine, pyridine, L-leucine. The top five metabolites in terms of abundancecollectively made up close to half (48\%) of the DHEA- FF metabolome (Figure 2A). In DHEA+, the FF metabolome profile of highly abundant metaboliteswas different, with cortisol as the most abundance metabolite, followed by L-alanine, L-phenylalanine, pyridine, L-isoleucine and L-leucine. These top six metabolites collectively made up 49.5\% of the DHEA+ FF metabolome (Figure 2B). Interestingly, pyridine, considered a non-endogenous metabolite (HMDB0000926)was found in such high abundance suggesting it came from the synthesis of DHEA.(42)The observed MS/MS spectra of pyridine at various eV matched very well with HMDB database (Figure S2), which suggested its correct identification.As a precursor to testosterone and estrogen, DHEA could be converted to testosterone, and aromatized to estrogen; in the case of POR, exogenous DHEA was proposed to increase androgens in promoting folliculogenesis and potentiate the effects of gonadotropins. $(8,43,44) \mathrm{FF}$ testosterone was detected in our metabolomics profiling, although the differences between DHEA+ and DHEA-group were small [DHEA-: mean signal intensity= 2294.5; DHEAt: mean signal intensity $=2267.75$ (testosterone), $p>0.05$; Figure S2B]. Next, we screened for prominent metabolites that are differential with DHEA treatment in POR using the criteria of $\beta$-coefficient regression values $>1.2$ and $p<0.05$. Among the FF metabolites, glycerophosphocholine, linoleic acid, progesterone, and L-valine fulfilled the screening criteria and were significantly lower in DHEA+ relative to DHEA- (Student's t-test, $p<0.05-0.005$; Figure 3AD).Although not significant, pregnenolone, a cholesterol metabolite and steroid that is upstream of DHEA metabolism, was detected only in DHEA+ (6/18 subjects), and not DHEA- (0/16 subjects). Receiver operating characteristic (ROC) analyses of the four metabolites revealed area under the curve (AUC) ranging from 0.711 (progesterone), 0.730 (glycerophosphocholine), 0.785 (linoleic acid) and 0.818 (Lvaline) ( $p<0.05-0.01$; Figure $3 \mathrm{E}-\mathrm{H})$, suggesting the plausible utility of these FF metabolites in monitoring DHEA treatment.Additionally, linoleic acid and L-valine remained significantly lower in DHEA+ $(p<0.05$, $p<0.001$ for both) when women with endometriosis $(N=5)$ were removed from analysis, strongly suggesting the significant effect of DHEA on these metabolites (Figure S3).

\section{FF cytokine profile in response to DHEA}

Of the 45 cytokines, chemokines and growth factors investigated, 22 were detected in human FF, comprising of10 cytokines (IFNy, IL12p70, IL13, IL1b, TNFa, IL1Ra, IL5, IL7, IL10, IL18), 6 chemokines (eotaxin, IP-10, MCP1, MIP1 $\beta$, SCF, SDF-1a) and 8 growth factors (bNGF, BDNF, EGF, HGF, LIF, PIGF, VEGFA, VEGF-D). Among them, FF MCP1, IFNy, LIF andVEGF-D were significant lower in DHEA+ compared to DHEA- $(p=0.03,0.014,0.031,0.0161$ respectively; Figure 4$)$. No correlation was found between the significant cytokines and metabolites.

\section{Discussion}

The follicular milieu provides oocytes with a specialized microenvironment that promotes the developmental competence of oocytes. This LC-MS/MS metabolomics study extends the human FF metabolome space in terms of characterization of its constituents, providing new insights into the complexities of oocyte development especially in POR women,(45) as well as with DHEA 
supplementation.(17-19,46,47)Due to the highly confident identification based on MS/MS, and mass accuracy of LC-MS/MS-based metabolomics, we were able to distinguish progesterone from DHEA, an advantage over interference-prone immunoassays that face a cross-reactivity bioanalytical problem.(48) Similarly, LC-MS/MS-based determination of androgens was preferred over immunoassays due to strong interference from DHEA.(49)We did not detect E1 and E2; because for phenolic hydroxyl group of estrogens to act as proton donors, the signal would be more sensitive in the negative ion mode electrospray ionization(50) than in the positive ion mode which was used in this study. Aside from previously reported constituents of FFsuch as linoleic acid,(17)amino acids,(18)and steroids including progesterone, testosterone(51), this study also captured metabolic products of ovarian steroidogenesis, cholesterols and glucocorticoids in the FF.

FF metabolomes distinguished DHEA+ from DHEA- The identification of four differential metabolites (glycerophosphocholine, linoleic acid, progesterone, and valine) in this study may alert us to the metabolic effects of exogenous DHEA supplementation and plausibly using them to achieve improved outcomes. (52-54)Choline and derivatives are an emerging class of metabolites critical in developmental competence of fertilized oocytes,(19) and glycerophosphorylcholine was found increased in the DHEA+ group. Glycerophosphorylcholine is formed from the breakdown of phosphatidylcholine, and is an organic osmolyte, plausibly affecting concentrations of other constitutes of $F F,(16)$ and regulation of the diffusion of compounds into FF necessary for folliculogenesis and oogenesis.(55)PORs are known to exhibit a low diffusion of exogenous gonadotropin into FF, which is correlated with poor IVF outcomes. (56)It is conceivable that DHEA induced the metabolism of phosphatidylcholine to glycerophosphorylcholine. Progesterone is one of the key hormones for the progress of the first metiotic division in oocyte maturation, but changes to progesterone levels with DHEA supplementation has been controversial (48). Our metabolomics study revealed for the first time that DHEA supplementation led to a decrease in FF progesterone levels, but to what impact lower progesterone induced by DHEA supplementation has on PORs remains to be investigated. Valine degradation has been previously reported in a proteomics study comparing competent versus incompetent buffalo oocyte proteome.(57)In a metabolomics study, valine metabolism was also identified in bovine cumulus and cumulus-oocytecomplex-conditioned media that undergo oocyte maturation;(58)although in both studies, valine was not directly detected in the omics profiling. In humans, degenerate oocytes or germinal vesicles that failed meiotically to reach metaphase II deplete valine more than competent oocytes, in other words lower valine levels in culture media which is consistent with our results, and suggest plausible biological roles of valine in oocyte maturation. Interestingly, we noted a segregation of DHEA+ patients with low and high level of valine, with the high valine group approaching concentrations of the DHEA- group. Together with valine's high AUC value, it is tempting to speculate that valine can be used as biomarker for monitoring individual DHEA supplementation.Linoleic acid is the most abundant polyunsaturated fatty acid in the bovine(59)and human FF (Figure 2), and varying concentrations of linoleic acid havereportedly different effects on oocyte maturation. At a concentration of $100 \mu \mathrm{M}$, linoleic acidadded to maturation media inhibitsbovine oocyte maturation and subsequent blastocyst development through increasing prostaglandin $E_{2}$ concentration in the medium, decreasing intracellular cAMP, decreasing phosphorylation 
of the MAPK1 and AKT and inhibited germinal vesicle breakdown.(59,60)Conversely, at concentrations at $50 \mu \mathrm{M}$ or below, linoleic acid improved oocyte quality by increasing the content of neutral lipids stored in lipid droplets.(60) Our metabolomics results suggest that FF linoleic acid can be another biomarker for titrating and monitoring individual DHEA supplementation. Therefore, given the observed trends of increased retrieved oocytes and metaphase II oocytes in DHEA+ group and published literature, our data suggest that DHEA might exert protective effects on oocytes in POR patients, albeit at an insufficient dose or treatment duration.

The elevated DHEA-sulphate levels coupled with a lack of difference in FF testosterone with DHEA supplementation suggest the following possibilities in POR patients: (i) inadequate DHEA conversion to testosterone due to polymorphism in SULT2A1, CYP19A1 and FMR1 genes,(61)or (ii) long CAG repeats in androgen receptor gene which is linked to itslower transcriptional activity at the promoters of genes involved in the metabolism of DHEA to testosterone.(62)The former is unlikely: in a case-control study involving 94 subjects, androgen secretion was not impaired in pre-ovulatory follicles of POR compared to normal responders, and similar levels of follicular testosterone levels was reported.(63)However, ethnicity and genetic predispositions might play a role as Chinese women are reported to have higher free androgens and African American women lower,(64) which might explain their differences in pregnancy rates in association with IVF than those observed among other ethnic groups. Conversely, long CAG repeats is associated with risk of PORand oocyte insensitivity to androgenic stimulation,(65)thus hinting a tenable rationale on the observed similar FF androgen levels between the DHEA- and DHEA+ subjects in this study. The abundance of cortisol in the DHEA+ subjects is interesting, in particular that DHEA reduces circulating cortisol,(66)indicating follicular versus systemic difference in how DHEA affects cortisol levels. In vitro, it was noted that DHEA suppresses cortisol activity,(67)including the antagonist effects of DHEA on the anti-inflammatory responses induced by cortisol via glucocorticoid receptor-mediated pathways.(68)It is noteworthy that high FF cortisol levels found in fertilized IVF individuals compared to unfertilized individuals led to the postulation that oocyte exposure to cortisol is required with oocyte maturation.(69)The higher levels of FF cortisol observed in DHEA+ subjects therefore argues for a compensatory response to modulate the ratio of the two hormonesin an attempt to maintain a favourable FF response to mature oocytes.(68)

In mouse models of polycystic ovary syndrome, treatment with DHEA resulted in increased production of cytokines such as serum TNFa, IL-6, IL12p70, and IFNy. $(70,71)$ In this study, DHEA supplementation lead to the reduction of FF IFNY, LIF, MCP-1, and VEGF-D levels. It appears that DHEA modulates chemokines and growth factors in POR FFwithout a clear Th1 or Th2 immune response as proposed.(54)LIF or leukemia inhibitory factor is expressed in the ovary and controls follicular growth.(72) It was reported thatLIF suppressed the growth of primary, secondary, and early antral follicles in cultured ovarian tissues. (73) The authors postulated that LIF produced in the late antral or graafian follicles is secreted to suppress the growth of the neighbouring primary, secondary, and early antral follicles as part of follicular growth.(16) Interestingly, when hCG is administered in rhesus macaques, at $12 \mathrm{~h}$ follicular LIF levels increase and induce follicle rupture and ovulation and decrease at $24 \mathrm{~h}$.(74)In our study, the number of MII oocytes and oocytes trended higher in the DHEA+ group, suggesting that the biological roles of LIF 
might have been achieved (follicular maturation and rupture) but inadequate to generate a clinically significant outcome. In vitro results suggested that follicles produce VEGF-A, with VEGF-A inducing the expanding vasculature to support the increased needs growing follicles.(75)The decrease in VEGF-A in DHEA+ individuals is intriguing. Fisher et al., described that in cultured follicles, the rise in VEGF-A levels in faster-growing follicles are dependent on FSH dose and oxygen tension.(76) There have been reports that DHEA inhibits oxygen consumption in neurons,(77)tempting the postulation that DHEA inhibited oxygen consumption in follicle that subsequently led to lower production of VEGF-A in DHEA+ individuals. Further, the lack of correlation between the significant cytokines and metabolites suggeststhat DHEA converting to steroids which subsequently modulate cytokine production within the follicular microenvironment is more complex than originally thought.

In conclusion, our study provided new insights to POR FF at the metabolome level, and as indicated from the FF metabolome analysis, exogenous DHEA to these patients altered the overall metabolome coverage and abundance to four metabolites. LC-MS global (untargeted) metabolomics analysis has provided the ability to reveal biologically relevant changes within a system, even at sensitive ranges before the precedence of gross morphological or phenotypical changes.(78)Hypotheses generated from this study included plausible mechanisms underlying DHEAmetabolism, and the potential utility of glycerophosphocholine, linoleic acid, progesterone, and L-valine as markers to assess DHEA supplementation. Therefore, future directions include targeted quantitative LC-MS/MS approaches to be developed to detect and quantify four "responder" metabolites in approaches similar to those previously conducted on human peritoneal fluids and sera.(79-81) to design treatment based on metabolomics profiles. Steroid hormones including testosterone should also be quantified via LC-MS/MS to establish baseline levels before commencing DHEA supplementation. Further, comparing POR and normal responders will provide further insights to the alteration of the FF metabolome, and reach a deeper understanding of underpinning pathophysiology to POR.

\section{Abbreviations}

dehydroepiandrosterone (DHEA);electrospray ionization (ESI);follicular fluid (FF);follicle stimulating hormone (FSH); human chorionic gonadotropin ( $\mathrm{hCG}$ ); in vitro fertilization (IVF);liquid chromatographytandem mass spectrometry (LC-MS/MS);poor ovarian responders (POR);partial least squaresdiscriminant regression (PLSR);quality control (QC);Principal Component Analysis (PCA)

\section{Declarations}

\section{- Ethical Approval and Consent to participate}

The local Institutional Review Board approved the study (CIRB/2011/404/D) and written informed consent was obtained from each participant.

\section{- Consent for publication}


Yes

\section{- Availability of supporting data}

Available upon request

- Competing interests

Not applicable

\section{- Funding}

This work was supported by SingHealth Foundation(SHF/CTG034/2010) to VVF, and the National Medical Research Council Centre Grant Programme (NMRC/CG/M003/2017) to YHL. JKYC received salary support from Singapore's Ministry of Health's National Medical Research Council (NMRC/CSA(SI)/008/2016).

\section{- Authors' contributions}

VVFand JCKY recruited patients. JZ performed metabolomics and cytokine analyses. DB performed statistical analysis. YT provided expert help on metabolomics. LYH and JCKY supervised the work and resources. All authors agreed to submission of the manuscript and have agreed to the order of authors.

\section{- Acknowledgements}

The authors would like to thank the patients for their participation and nurses for their frontline service.

\section{- Disclosure of conflict-of-interest statement}

The authors have nothing to disclose.

\section{References}

1. Surrey ES, Schoolcraft WB. Evaluating strategies for improving ovarian response of the poor responder undergoing assisted reproductive techniques. Fertil. Steril. 2000;73(4):667-676.

2. Devine K, Mumford SL, Wu M, DeCherney AH, Hill MJ, Propst A. Diminished ovarian reserve in the United States assisted reproductive technology population: diagnostic trends among 181,536 cycles from the Society for Assisted Reproductive Technology Clinic Outcomes Reporting System. Fertil. Steril. 2015;1109. doi:10.1016/j.fertnstert.2015.05.017.

3. Ferraretti AP, La Marca A, Fauser BCJM, Tarlatzis B, Nargund G, Gianaroli L, ESHRE working group on Poor Ovarian Response Definition. ESHRE consensus on the definition of "poor response" to ovarian stimulation for in vitro fertilization: the Bologna criteria. Hum. Reprod. 2011;26(7):1616-24.

4. Kolibianakis EM, Venetis CA, Diedrich K, Tarlatzis BC, Griesinger G. Addition of growth hormone to gonadotrophins in ovarian stimulation of poor responders treated by in-vitro fertilization: A 
systematic review and meta-analysis. Hum. Reprod. Update 2009;15(6):613-622.

5. Haning R V, Flood CA, Hackett RJ, Loughlin JS, McClure N, Longcope C. Metabolic clearance rate of dehydroepiandrosterone sulfate, its metabolism to testosterone, and its intrafollicular metabolism to dehydroepiandrosterone, androstenedione, testosterone, and dihydrotestosterone in vivo. J. Clin. Endocrinol. Metab. 1991;72(5):1088-95.

6. Casson PR, Santoro N, Elkind-Hirsch K, Carson SA, Hornsby PJ, Abraham G, Buster JE. Postmenopausal dehydroepiandrosterone administration increases free insulin-like growth factor-I and decreases high-density lipoprotein: A six- month trial. Fertil. Steril. 1998;70(1):107-110.

7. Barad D, Gleicher N. Effect of dehydroepiandrosterone on oocyte and embryo yields, embryo grade and cell number in IVF. Hum. Reprod. 2006;21(11):2845-2849.

8. Casson PR. Dehydroepiandrosterone supplementation augments ovarian stimulation in poor responders: a case series. Hum. Reprod. 2000;15(10):2129-2132.

9. Burger HG. Androgen production in women. Fertil. Steril. 2002;77(4):S3-S5.

10. Roy S, Mahesh VB, Greenblatt RB. Effect of dehydroepiandrosterone and 84 -androstenedione on the reproductive organs of female rats: Production of cystic changes in the ovary. Nature 1962;196(4849):42-43.

11. Broekmans FJ, Soules MR, Fauser BC. Ovarian Aging: Mechanisms and Clinical Consequences. Endocr. Rev. 2009;30(5):465-493.

12. Barad D, Brill H, Gleicher N. Update on the use of dehydroepiandrosterone supplementation among women with diminished ovarian function. In: Journal of Assisted Reproduction and Genetics.Vol 24.; 2007:629-634.

13. Kyrou D, Kolibianakis EM, Venetis CA, Papanikolaou EG, Bontis J, Tarlatzis BC. How to improve the probability of pregnancy in poor responders undergoing in vitro fertilization: a systematic review and meta-analysis. Fertil. Steril. 2009;91(3):749-766.

14. Bächler M, Menshykau D, De Geyter C, Iber D. Species-specific differences in follicular antral sizes result from diffusion-based limitations on the thickness of the granulosa cell layer. MHR Basic Sci. Reprod. Med. 2014;20(3):208-221.

15. Seli E, Babayev E, Collins SC, Nemeth G, Horvath TL. Minireview: Metabolism of Female Reproduction: Regulatory Mechanisms and Clinical Implications. Mol. Endocrinol. 2014;28(6):790804.

16. Rodgers RJ, Irving-Rodgers HF. Formation of the ovarian follicular antrum and follicular fluid. Biol. Reprod. 2010;82(6):1021-9.

17. O'Gorman A, Wallace M, Cottell E, Gibney MJ, McAuliffe FM, Wingfield M, Brennan L. Metabolic profiling of human follicular fluid identifies potential biomarkers of oocyte developmental competence. Reproduction 2013;146(4):389-395.

18. Lan Xia, Zhao X, Sun Y, Hong Y, Yuping Gao SH. Metabolomic profiling of human follicular fluid from patients with repeated failure of in vitro fertilization using gas chromatography/mass spectrometry. Int. J. Clin. Experimenal Pathol. 2014;7(10):7220-7229. 
19. Wallace M, Cottell E, Gibney MJ, McAuliffe FM, Wingfield M, Brennan L. An investigation into the relationship between the metabolic profile of follicular fluid, oocyte developmental potential, and implantation outcome. Fertil. Steril. 2012;97(5):1078-1084.e8.

20. Piñero-Sagredo E, Nunes S, de los Santos MJ, Celda B, Esteve V. NMR metabolic profile of human follicular fluid. NMR Biomed. 2010;23(5):485-495.

21. Ban Y, Ran H, Chen Y, Ma L. Lipidomics analysis of human follicular fluid form normal-weight patients with polycystic ovary syndrome: a pilot study. J. Ovarian Res. 2021;14(1):135.

22. Zamah AM, Hassis ME, Albertolle ME, Williams KE. Proteomic analysis of human follicular fluid from fertile women. Clin. Proteomics 2015;12(1):5.

23. Angelucci S, Ciavardelli D, Di Giuseppe F, Eleuterio E, Sulpizio M, Tiboni GM, Giampietro F, Palumbo P, Di llio C. Proteome analysis of human follicular fluid. Biochim. Biophys. Acta - Proteins Proteomics 2006;1764(11):1775-1785.

24. Jarkovska K, Martinkova J, Liskova L, Halada P, Moos J, Rezabek K, Gadher SJ, Kovarova H.

Proteome Mining of Human Follicular Fluid Reveals a Crucial Role of Complement Cascade and Key Biological Pathways in Women Undergoing in Vitro Fertilization. J. Proteome Res. 2010;9(3):12891301.

25. Hanrieder J, Nyakas A, Naessén T, Bergquist J. Proteomic Analysis of Human Follicular Fluid Using an Alternative Bottom-Up Approach. J. Proteome Res. 2008;7(1):443-449.

26. Estes SJ, Ye B, Qiu W, Cramer D, Hornstein MD, Missmer SA. A proteomic analysis of IVF follicular fluid in women $\leq 32$ years old. Fertil. Steril. 2009;92(5):1569-1578.

27. Chen F, Spiessens C, D'Hooghe T, Peeraer K, Carpentier S. Follicular fluid biomarkers for human in vitro fertilization outcome: Proof of principle. Proteome Sci. 2016;14(1):17.

28. Li C-J, Lin L-T, Tsui K-H. Dehydroepiandrosterone Shifts Energy Metabolism to Increase Mitochondrial Biogenesis in Female Fertility with Advancing Age. Nutrients 2021;13(7):2449.

29. Yakin K, Urman B. DHEA as a miracle drug in the treatment of poor responders; hype or hope? Hum. Reprod. 2011;26(8):1941-1944.

30. PG A, Simi G, Ruggiero M, Pinelli S, OM DB, Papini F, Papini S, Monteleone P, Cela V. DHEA supplementation improves follicular microenviroment in poor responder patients. Gynecol. Endocrinol. 2012;28(9):669-673.

31. Yeung TWY, Chai J, Li RHW, Lee VCY, Ho PC, Ng EHY. A randomized, controlled, pilot trial on the effect of dehydroepiandrosterone on ovarian response markers, ovarian response, and in vitro fertilization outcomes in poor responders. Fertil. Steril. 2014;102(1):108-115.e1.

32. Kara M, Aydin T, Aran T, Turktekin N, Ozdemir B. Does dehydroepiandrosterone supplementation really affect IVF-ICSI outcome in women with poor ovarian reserve? Eur. J. Obstet. Gynecol. Reprod. Biol. 2014;173:63-5.

33. Chern C-U, Tsui K-H, Vitale SG, Chen S-N, Wang P-H, Cianci A, Tsai H-W, Wen Z-H, Lin L-T. Dehydroepiandrosterone (DHEA) supplementation improves in vitro fertilization outcomes of poor 
ovarian responders, especially in women with low serum concentration of DHEA-S: a retrospective cohort study. Reprod. Biol. Endocrinol. 2018;16(1):90.

34. Chen S-N, Tsui K-H, Wang P-H, Chern C-U, Wen Z-H, Lin L-T. Dehydroepiandrosterone Supplementation Improves the Outcomes of in vitro Fertilization Cycles in Older Patients With Diminished Ovarian Reserve. Front. Endocrinol. (Lausanne). 2019;10. doi:10.3389/fendo.2019.00800.

35. Hazeldine J, Arlt W, Lord JM. Dehydroepiandrosterone as a regulator of immune cell function. J. Steroid Biochem. Mol. Biol. 2010;120(2-3):127-136.

36. Mason HD, Margara R, Winston RM, Seppala M, Koistinen R, Franks S. Insulin-like growth factor-I (IGF-I) inhibits production of IGF-binding protein-1 while stimulating estradiol secretion in granulosa cells from normal and polycystic human ovaries. J. Clin. Endocrinol. Metab. 1993;76(5):1275-1279.

37. Lee YH, Goh WW Bin, Ng CK, Raida M, Wong L, Lin Q, Boelsterli U a, Chung MCM. Integrative toxicoproteomics implicates impaired mitochondrial glutathione import as an off-target effect of troglitazone. J. Proteome Res. 2013;12(6):2933-45.

38. Cui L, Fang J, Ooi EE, Lee YH. Serial Metabolome Changes in a Prospective Cohort of Subjects with Influenza Viral Infection and Comparison with Dengue Fever. J. Proteome Res. 2017;16(7):26142622.

39. Harden SL, Zhou J, Gharanei S, Diniz-da-Costa M, Lucas ES, Cui L, Murakami K, Fang J, Chen Q, Brosens JJ, Lee YH. Exometabolomic Analysis of Decidualizing Human Endometrial Stromal and Perivascular Cells. Front. Cell Dev. Biol. 2021;9. doi:10.3389/fcell.2021.626619.

40. Zhou J, Chern BSM, Barton-Smith P, Phoon JWL, Tan TY, Viardot-Foucault V, Ku CW, Tan HH, Chan JKY, Lee YH. Peritoneal Fluid Cytokines Reveal New Insights of Endometriosis Subphenotypes. Int. J. Mol. Sci. 2020;21(10):3515.

41. Wishart DS, Tzur D, Knox C, Eisner R, Guo AC, Young N, Cheng D, Jewell K, Arndt D, Sawhney S, Fung C, Nikolai L, Lewis M, Coutouly M-A, Forsythe I, Tang P, Shrivastava S, Jeroncic K, Stothard P, Amegbey G, Block D, Hau DD, Wagner J, Miniaci J, Clements M, Gebremedhin M, Guo N, Zhang Y, Duggan GE, Macinnis GD, Weljie AM, Dowlatabadi R, Bamforth F, Clive D, Greiner R, Li L, Marrie T, Sykes BD, Vogel HJ, Querengesser L. HMDB: the Human Metabolome Database. Nucleic Acids Res. 2007;35(Database issue):D521-6.

42. Williams JR, Boehm JC. Studies on the synthesis of dehydroepiandrosterone (DHEA) phosphatide. Steroids 1995;60(4):333-6.

43. Nielsen ME, Rasmussen IA, Kristensen SG, Christensen ST, Mollgard K, Wreford Andersen E, Byskov AG, Yding Andersen C. In human granulosa cells from small antral follicles, androgen receptor mRNA and androgen levels in follicular fluid correlate with FSH receptor mRNA. Mol. Hum. Reprod. 2011;17(1):63-70.

44. Gleicher N, Weghofer A, Barad DH. The role of androgens in follicle maturation and ovulation induction: friend or foe of infertility treatment? Reprod. Biol. Endocrinol. 2011;9(1):116. 
45. Emori MM, Drapkin R. The hormonal composition of follicular fluid and its implications for ovarian cancer pathogenesis. Reprod. Biol. Endocrinol. 2014;12(1):60.

46. Bedaiwy M, Shahin AY, AbulHassan AM, Goldberg JM, Sharma RK, Agarwal A, Falcone T. Differential expression of follicular fluid cytokines: relationship to subsequent pregnancy in IVF cycles. Reprod. Biomed. Online 2007;15(3):321-325.

47. Baskind NE, Orsi NM, Sharma V. Follicular-phase ovarian follicular fluid and plasma cytokine profiling of natural cycle in vitro fertilization patients. Fertil. Steril. 2014;102(2):410-8.

48. Forman EJ, Franasiak JM, Scott RT. Elevated progesterone levels in women on DHEA supplementation likely represent assay interference. J. Assist. Reprod. Genet. 2015;32(4):661-661.

49. Heald AH, Butterworth A, Kane JW, Borzomato J, Taylor NF, Layton T, Kilpatrick ES, Rudenski A. Investigation into possible causes of interference in serum testosterone measurement in women. Ann. Clin. Biochem. 2006;43(3):189-195.

50. Guo T, Gu J, Soldin OP, Singh RJ, Soldin SJ. Rapid measurement of estrogens and their metabolites in human serum by liquid chromatography-tandem mass spectrometry without derivatization. Clin. Biochem. 2008;41(9):736-741.

51. Wen X, Li D, Tozer AJ, Docherty SM, lles RK. Estradiol, progesterone, testosterone profiles in human follicular fluid and cultured granulosa cells from luteinized pre-ovulatory follicles. Reprod. Biol. Endocrinol. 2010;8(1):117.

52. Wiser A, Gonen O, Ghetler Y, Shavit T, Berkovitz A, Shulman A. Addition of dehydroepiandrosterone (DHEA) for poor-responder patients before and during IVF treatment improves the pregnancy rate: A randomized prospective study. Hum. Reprod. 2010;25(10):2496-2500.

53. Hu Q, Hong L, Nie M, Wang Q, Fang Y, Dai Y, Zhai Y, Wang S, Yin C, Yang X. The effect of dehydroepiandrosterone supplementation on ovarian response is associated with androgen receptor in diminished ovarian reserve women. J. Ovarian Res. 2017;10(1):32.

54. Zhang J, Qiu X, Gui Y, Xu Y, Li D, Wang L. Dehydroepiandrosterone improves the ovarian reserve of women with diminished ovarian reserve and is a potential regulator of the immune response in the ovaries. Biosci. Trends 2015;9(6):350-359.

55. Hsueh AJW, Kawamura K, Cheng Y, Fauser BCJM. Intraovarian Control of Early Folliculogenesis. Endocr. Rev. 2015;36(1):1-24.

56. Nagata Y, Honjou K, Sonoda M, Sumii Y, Inoue Y, Kawarabayashi T. Pharmacokinetics of exogenous gonadotropin and ovarian response in in vitro fertilization. Fertil. Steril. 1999;72(2):235-239.

57. Chen L, Zhai L, Qu C, Zhang C, Li S, Wu F, Qi Y, Lu F, Xu P, Li X, Shi D. Comparative Proteomic Analysis of Buffalo Oocytes Matured in vitro Using iTRAQ Technique. Sci. Rep. 2016;6(1):31795.

58. Uhde K, van Tol HTA, Stout TAE, Roelen BAJ. Metabolomic profiles of bovine cumulus cells and cumulus-oocyte-complex-conditioned medium during maturation in vitro. Sci. Rep. 2018;8(1):9477.

59. Marei WF, Wathes DC, Fouladi-Nashta AA. Impact of linoleic acid on bovine oocyte maturation and embryo development. Reproduction 2010;139(6):979-988. 
60. Carro M, Buschiazzo J, Ríos GL, Oresti GM, Alberio RH. Linoleic acid stimulates neutral lipid accumulation in lipid droplets of maturing bovine oocytes. Theriogenology 2013;79(4):687-694.

61. Shohat-Tal A, Sen A, Barad DH, Kushnir V, Gleicher N. Genetics of androgen metabolism in women with infertility and hypoandrogenism. Nat. Rev. Endocrinol. 2015;11(7):429-441.

62. Borgbo T, Macek M, Chrudimska J, Jeppesen JV, Hansen LL, Andersen CY. Size matters: Associations between the androgen receptor CAG repeat length and the intrafollicular hormone milieu. Mol. Cell. Endocrinol. 2016;419:12-17.

63. de los Santos MJ, García-Laez V, Beltrán D, Labarta E, Zuzuarregui JL, Alamá P, Gámiz P, Crespo J, Bosch E, Pellicer A. The follicular hormonal profile in low-responder patients undergoing unstimulated cycles: Is it hypoandrogenic? Hum. Reprod. 2013;28(1):224-9.

64. Sutton-Tyrrell K. Sex Hormone-Binding Globulin and the Free Androgen Index Are Related to Cardiovascular Risk Factors in Multiethnic Premenopausal and Perimenopausal Women Enrolled in the Study of Women Across the Nation (SWAN). Circulation 2005;111(10):1242-1249.

65. Lledo B, Llácer J, Ortiz JA, Martinez B, Morales R, Bernabeu R. A pharmacogenetic approach to improve low ovarian response: The role of CAG repeats length in the androgen receptor gene. Eur. J. Obstet. Gynecol. Reprod. Biol. 2018;227:41-45.

66. Kroboth PD, Amico JA, Stone RA, Folan M, Frye RF, Kroboth FJ, Bigos KL, Fabian TJ, Linares AM, Pollock BG, Hakala C. Influence of DHEA Administration on 24-Hour Cortisol Concentrations. J. Clin. Psychopharmacol. 2003;23(1):96-99.

67. Buoso E, Lanni C, Molteni E, Rousset F, Corsini E, Racchi M. Opposing effects of cortisol and dehydroepiandrosterone on the expression of the receptor for Activated C Kinase 1: Implications in immunosenescence. Exp. Gerontol. 2011;46(11):877-883.

68. Kamin HS, Kertes DA. Cortisol and DHEA in development and psychopathology. Horm. Behav. 2017;89:69-85.

69. Keay SD. Higher cortisol:cortisone ratios in the preovulatory follicle of completely unstimulated IVF cycles indicate oocytes with increased pregnancy potential. Hum. Reprod. 2002;17(9):2410-2414.

70. Sander V, Luchetti CG, Solano ME, Elia E, Di Girolamo G, Gonzalez C, Motta AB. Role of the N, N'dimethylbiguanide metformin in the treatment of female prepuberal BALB/c mice hyperandrogenized with dehydroepiandrosterone. Reproduction 2006;131(3):591-602.

71. Solano ME, Sander VA, Ho H, Motta AB, Arck PC. Systemic inflammation, cellular influx and upregulation of ovarian VCAM-1 expression in a mouse model of polycystic ovary syndrome (PCOS). J. Reprod. Immunol. 2011;92(1-2):33-44.

72. Abir R, Fisch B, Jin S, Barnnet M, Freimann S, Van den Hurk R, Feldberg D, Nitke S, Krissi H, Ao A. Immunocytochemical detection and RT-PCR expression of leukaemia inhibitory factor and its receptor in human fetal and adult ovaries. Mol. Hum. Reprod. 2004. doi:10.1093/molehr/gah047.

73. Komatsu K, Koya T, Wang J, Yamashita M, Kikkawa F, Iwase A. Analysis of the Effect of Leukemia Inhibitory Factor on Follicular Growth in Cultured Murine Ovarian Tissue. Biol. Reprod. 2015;93(1). doi:10.1095/biolreprod.115.128421. 
74. Murphy MJ, Halow NG, Royer PA, Hennebold JD. Leukemia Inhibitory Factor Is Necessary for Ovulation in Female Rhesus Macaques. Endocrinology 2016;157(11):4378-4387.

75. Xu J, Bernuci MP, Lawson MS, Yeoman RR, Fisher TE, Zelinski MB, Stouffer RL. Survival, growth, and maturation of secondary follicles from prepubertal, young, and older adult rhesus monkeys during encapsulated three-dimensional culture: Effects of gonadotropins and insulin. Reproduction 2010. doi:10.1530/REP-10-0284.

76. Fisher TE, Molskness TA, Villeda A, Zelinski MB, Stouffer RL, Xu J. Vascular endothelial growth factor and angiopoietin production by primate follicles during culture is a function of growth rate, gonadotrophin exposure and oxygen milieu. Hum. Reprod. 2013;28(12):3263-3270.

77. Safiulina D, Peet N, Seppet E, Zharkovsky A, Kaasik A. Dehydroepiandrosterone Inhibits Complex I of the Mitochondrial Respiratory Chain and is Neurotoxic In Vitro and In Vivo at High Concentrations. Toxicol. Sci. 2006;93(2):348-356.

78. Cui L, Lu H, Lee YH. Challenges and emergent solutions for LC-MS/MS based untargeted metabolomics in diseases. Mass Spectrom. Rev.2018;37(6):772-792.

79. Lee YH, Yang JX, Allen JC, Tan CS, Chern BSM, Tan TY, Tan HH, Mattar CNZ, Chan JKY. Elevated peritoneal fluid ceramides in human endometriosis-associated infertility and their effects on mouse oocyte maturation. Fertil. Steril. 2018;110(4):767-777.e5.

80. Lee YH, Tan CW, Venkatratnam A, Tan CS, Cui L, Loh SF, Griffith L, Tannenbaum SR, Chan JKY. Dysregulated sphingolipid metabolism in endometriosis. J. Clin. Endocrinol. Metab. 2014;99(10):E1913-21.

81. Lee YH, Cui L, Fang J, Chern BSM, Tan HH, Chan JKY. Limited value of pro-inflammatory oxylipins and cytokines as circulating biomarkers in endometriosis - a targeted 'omics study. Sci. Rep. 2016;6:26117.

\section{Tables}

Tables are available in the supplemental file section.

\section{Figures}




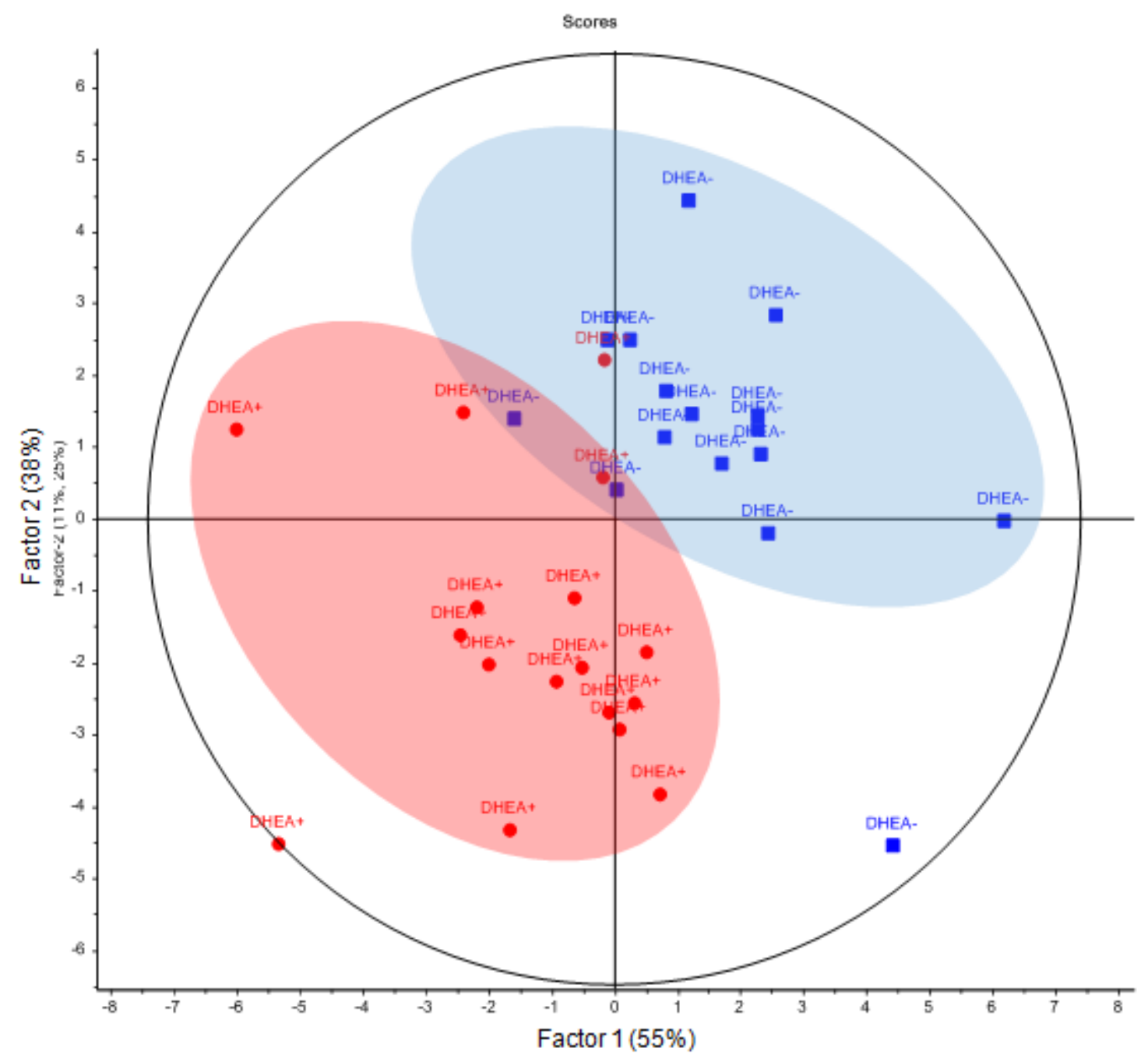

Figure 1

Partial Least Squares Scores plot of DHEA- and DHEA+ follicular fluid metabolome. Metabolomic data was median centred, and scaled by division with the standard deviation. The follicular fluid metabolome distinguished POR subjects on DHEA supplementation (DHEA+, red) and without DHEA supplementation (DHEA-, blue). 


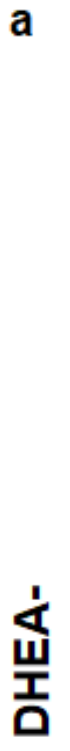

11a Hydraxyprogeszy on

L-Methiorine

Cresfinine
Valine

Alpha Aspraty-lysine

36-Hydary-5-chilenoic asid.

Dimethyl sulfore

Pipecolic soid =

Decarycholic acid gyoine corjugale

Lyeine- byeine1.

Pregnerolone

Cressinal N-Acetyl-L-alsine

D-Glutaminel-Glutamine
PC(18:1/9Z) 18:1(15Z))

CorvexdoneCoricosier one

Baide acidWacoeric acid

30 -Hydraxy-5-chilencic soid

16. Delindraprogesterone

Alpha N-pherylasongl-L-gl utamine

PC (18:1(9Z) 18:1(17Z))

Sphingosine

811 14. Eicosstrienoic acid

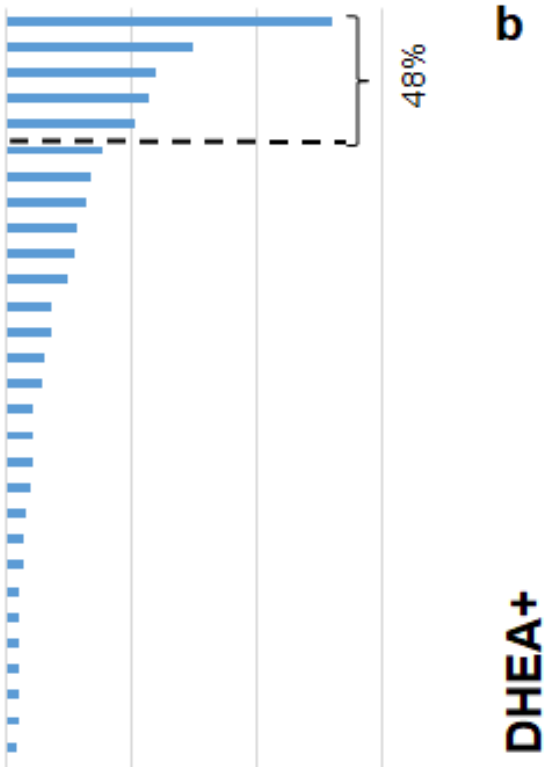

$100000200000 \quad 300000$

Intensity counts

b

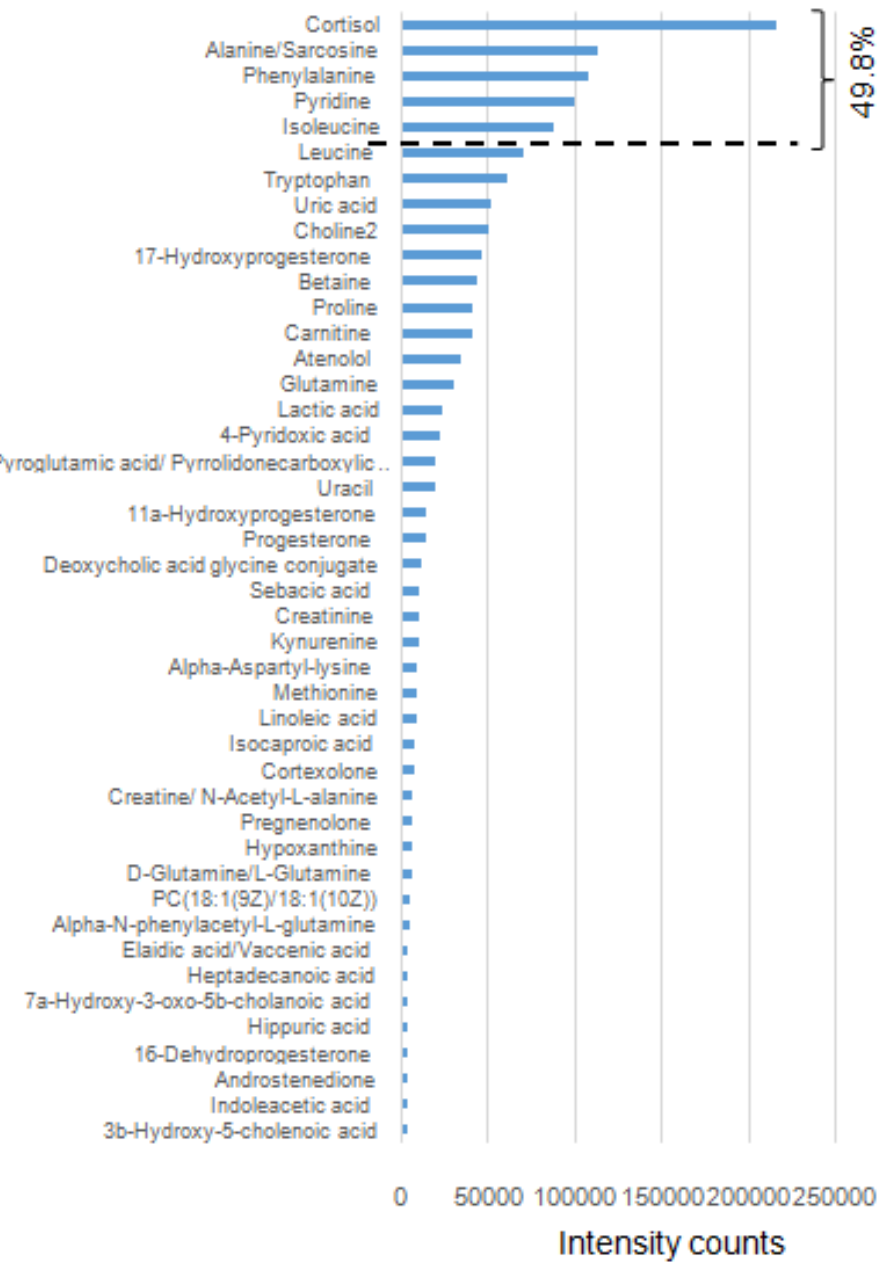

Figure 2

Histogram of follicular fluid metabolites in poor ovarian responders with and without DHEA supplementation. Follicular metabolome coverage and metabolite abundance as quantified by untargeted LC-MS/MS metabolomics in (A) DHEA- and (B) DHEA+ subjects, and were ranked according to their intensity counts. The top 50\% metabolites are shown, with progesterone being the major differentiating metabolite between the DHEA- and DHEA+ subjects. 
a

Linoleic acid

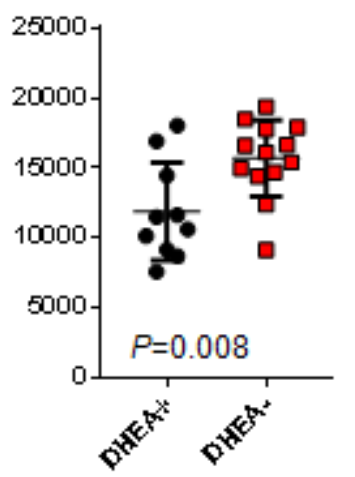

c

L-Valine

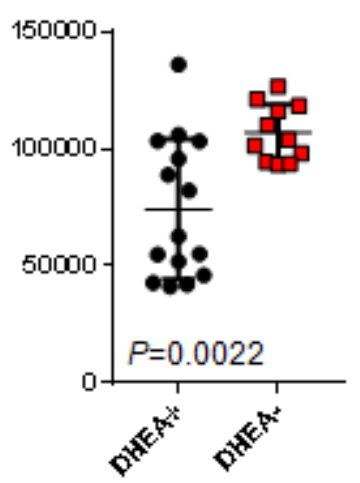

b

Progesterone

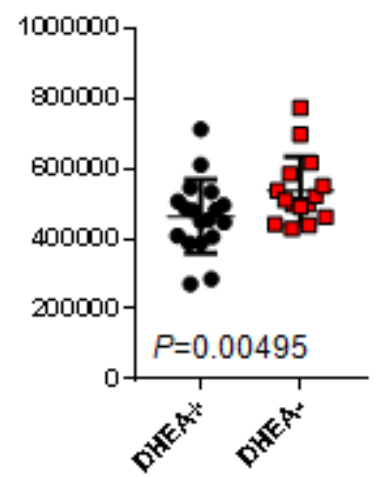

d

Glycerophosphocholine e

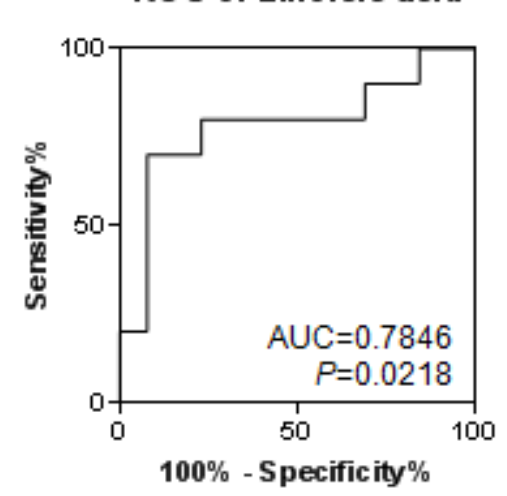

g

\section{e}

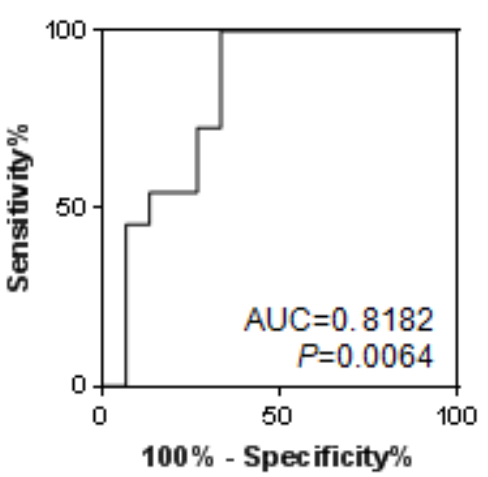

f
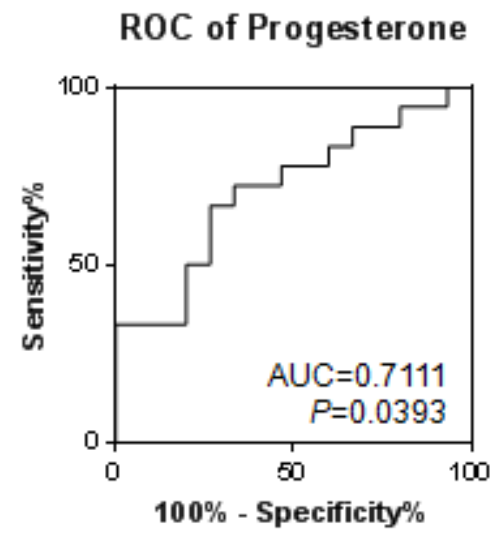

h ROC of Glycerophosphocholine

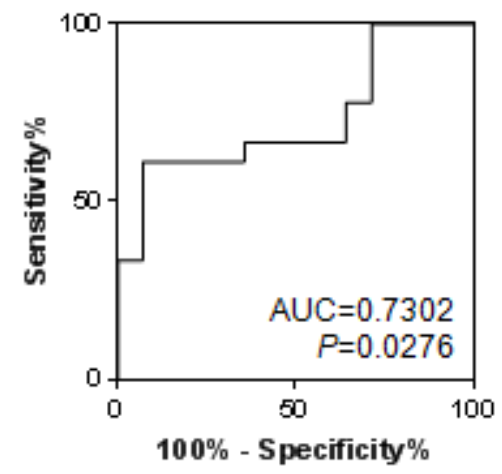

Figure 3

Significantly changed follicular fluid metabolites in DHEA+ and DHEA-patients. (A-D) Dot plots of significantly changed metabolites in poor ovarian responder patients. Student's t-tests were performed and $p<0.05$ is considered statistically significant. $(E-F)$ Corresponding receiver operating curve (ROC) analyses of the metabolites. Area under curve (AUC) of the metabolites and their P-values are reported.

A

MCP1

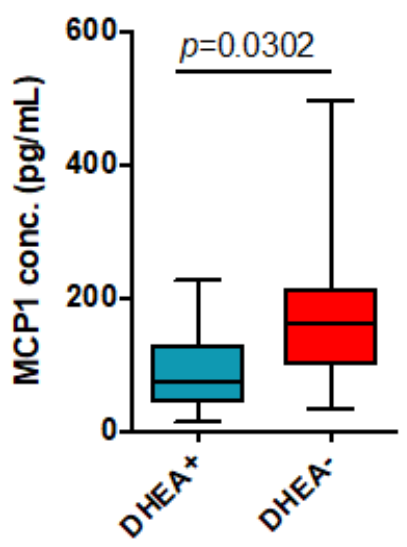

IFN $\gamma$

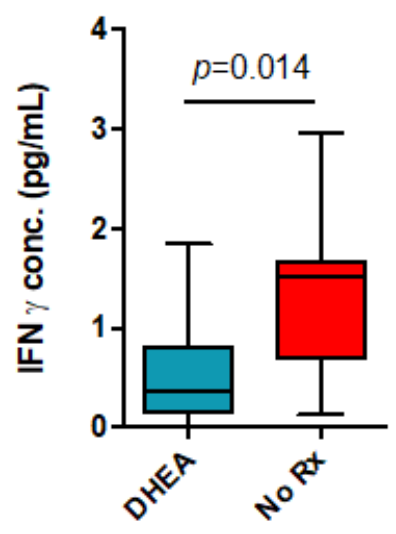

C

LIF

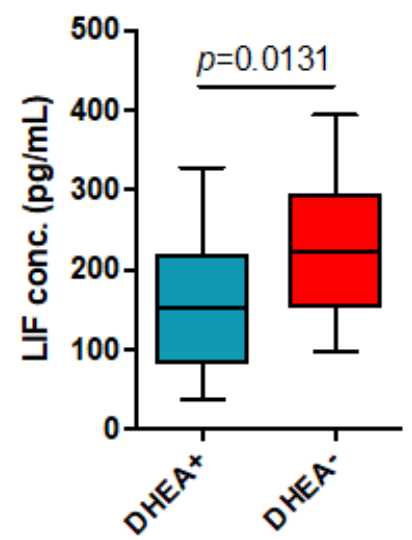

VEGF-D

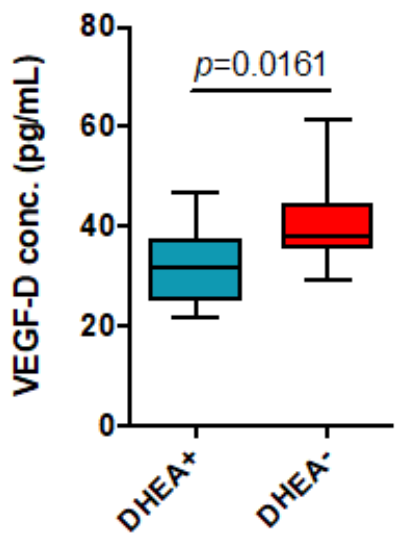

Figure 4 
Significantly changed follicular fluid cytokines in DHEA+ and DHEA-patients. Among 45 cytokines, chemokines and growth factors measured by multiplex immunoassay, (A) MCP-1, (B) IFNg, (C) LIF and (D) VEGF-D were significantly lower in POR subjects with DHEA supplementation. Student's t-tests were performed and $p<0.05$ is considered statistically significant.

\section{Supplementary Files}

This is a list of supplementary files associated with this preprint. Click to download.

- SupplementaryFiguresVVFJLetalFFMetabolome.pptx

- Table2.xlsx

- Table4.xlsx

- Table3.xlsx

- Table1.xlsx 